\section{IL-20's osteoporosis connection}

\section{By Michael J. Haas, Senior Writer}

A group of Taiwanese researchers has shown that an antibody against IL-20 helped treat osteoporosis in mice. ${ }^{1}$ The findings have grabbed the attention of Novo Nordisk A/S, which has an anti-IL-20 mAb in Phase II testing to treat rheumatoid arthritis and is now considering exploring the bone loss indication.

IL-20, a member of the IL-10 family of proinflammatory cytokines, is expressed in monocytes, epithelial cells and endothelial cells. The team's previous studies showed that IL-20 was upregulated in mouse models of atherosclerosis ${ }^{2}$ and that an antibody against the cytokine reduced brain infarct size in mouse models of ischemic stroke. ${ }^{3}$

In 2006, the team showed that blocking IL-20 with a soluble form of its receptor, IL-20 receptor- $\alpha$ (IL20RA; IL20R1), decreased disease severity in mouse models of RA. Surprisingly, the therapeutic protected the animals against bone destruction as well. ${ }^{4}$ Those data led the team to speculate that IL-20 played a role in pathological bone loss and that inhibiting it could help treat osteoporosis.

In a new paper in The Journal of Experimental Medicine, the team has reported that serum levels of IL-20 were greater in patients with osteopenia (low bone mineral density) and osteoporosis than in healthy controls. Similar results were seen in mouse models of ovariectomy-induced osteoporosis.

Teasing out the mechanism, the group found that IL-20 regulated signaling between two proteins-tumor necrosis factor receptor superfamily member 11a (TNFRSF11A; RANK; CD265), which is expressed on osteoclasts, and receptor activator of NF- $\mathrm{\kappa B}$ ligand (RANKL; TNFSF11), which is expressed by osteoblasts. The receptor-ligand pair is involved in the formation and differentiation of osteoclasts.

Il-20 itself increased the expression of Rank and Rankl in mouse osteoclast precursor cells and preosteoblast cells, respectively, whereas an anti-IL-20 antibody lowered expression in those respective cell types. The team also showed that Rank and Rankl were downregulated in Il20ra-deficient mice compared with in wild-type controls.

Lastly, the team showed that the anti-IL-20 antibody reduced bone loss and increased bone mineral density compared with an inactive control antibody in mouse models of osteoporosis.

Ming-Shi Chang, director and professor of biochemistry at National Cheng Kung University (NCKU), led the team. Chang was previously at Amgen Inc., where she was part of the team that discovered TNFRSF11B (OPG) - a RANKL scavenger and decoy-and showed that transgenic expression of OPG increased bone mineral density in healthy mice and decreased bone loss in rat models of osteoporosis. ${ }^{5}$ According to Chang, those findings eventually led the company to identify RANKL as an osteoporosis target and to develop denosumab, a mAb against RANKL, to treat the indication.

Amgen, Daiichi Sankyo Co. Ltd. and GlaxoSmithKline plc market Prolia/Xgeva denosumab to treat postmenopausal osteoporosis. The antibody is approved to prevent skeletal-related events in patients with bone metastases from solid tumors and to treat bone loss associated with hormone ablation in men with prostate cancer.

"IL-20 intrigues us as an osteoporosis target," said Mads Krogsgaard Thomsen, EVP and CSO of Novo Nordisk. "If an anti-IL-20 antibody could preserve bone tissue as well as cartilage in rheumatoid arthritis, that would be a positive effect."

Thomsen also told SciBX that Novo Nordisk's collaborator at Tokyo Medical University has "obtained similar results for our anti-IL-20 mAb in ovariectomized mouse models of osteoporosis."

He added that the company has not decided whether to begin a program in osteoporosis.

"I think the anti-IL-20 antibody will be good for any disease involving bone destruction, and I have confidence that it has many advantages over anti-RANKL antibodies." -Ming-Shi Chang,

\section{Bones to pick}

\section{National Cheng Kung University} IL-20's role in regulating osteoblast function during bone resorption. Chang declined to disclose the results but said the anti-IL-20 antibody's dual effect on osteoclast and osteoblast activity gave it an advantage over anti-RANKL antibodies, which affect only osteoclasts.

Additionally, she said the team has shown that the anti-IL-20 antibody reduced bone metastases in animal models of cancer. Results of that study will be the subject of a forthcoming paper, she said.

"I think the anti-IL-20 antibody will be good for any disease involving bone destruction, and I have confidence that it has many advantages over anti-RANKL antibodies," Chang told SciBX.

Josefin-Beate Holz, CMO of Ablynx N.V., was less sanguine about IL-20 inhibition and said the findings in the paper did not make it clear that IL-20 was a major driver in osteoporosis.

For example, Holz said the paper showed that mean serum levels of IL-20 were increased in some osteopenia and osteoporosis patients, but about $40 \%$ had IL-20 levels in the normal range.

She also noted that neither healthy control mice receiving the anti-IL-20 antibody nor young Il20ra-deficient mouse models of osteoporosis experienced an increase in bone mineral densitysuggesting that IL-20 is not always involved in normal or pathological bone resorption.

Chang countered that her team's previous studies showed that IL-20 activity is upregulated only in diseased states and not under normal physiological conditions. Thus, the antibody had no effect in healthy control mice because they lacked a pathological condition-such as the 


\section{ANALYSIS}

ovariectomy-related estrogen deficiency of the mouse models-that might upregulate IL-20, she said.

She also acknowledged it would be worthwhile to explore the possibility that estrogen regulates mechanisms of IL-20 expression.

Holz also said that "how IL-20's regulation of RANK and RANKL affects the balance between bone formation and bone degradation in humans remains to be seen. It is conceivable that IL-20 as a target to treat osteoporosis is too distant from the actual disease process for an antibody against it to have a robust effect. I think we would need to see the antibody's effects confirmed in nonhuman primates or other higher-order species."

Ablynx's ALX-0141, a Nanobody against RANKL, is in Phase I testing to prevent bone loss in postmenopausal osteoporosis, metastatic cancer and other indications.

"IL-20 is a cytokine still in need of further research," Thomsen said. "I would like to know more about the biology and pathobiology of IL-20, its mechanism of action, and which cell types express the cytokine and its receptor and when."

Holz also pointed out that chronic IL-20 inhibition could have significant immune side effects. "The IL-10 family cytokines have indispensable functions in innate immune responses to infection," and treating osteoporosis with an anti-IL-20 antibody could adversely affect those responses, she said.

Additionally, IL-20 inhibition might compromise immune system surveillance of primary and metastatic tumor cells.

Chang said her team has already conducted safety studies of the anti-IL-20 antibody in healthy mice and rats and has seen no adverse effects. Likewise, the team has observed no significant adverse phenotypes in Il20ra-deficient mice, further suggesting that blocking cytokine-receptor signaling with the antibody is safe, she said. But she added that a comprehensive pharmacokinetic study of the antibody would be required.

Thomsen added that Novo Nordisk's 40 -week toxicity studies of its anti-IL-20 mAb in macaques resulted in no safety issues.

NCKU holds patents covering use of the Chang team's anti-IL-20 antibody to treat osteoporosis, stroke and rheumatoid arthritis (RA). The IP is available for licensing or partnering, Chang said.

Haas, M.J. SciBX 4(34); doi:10.1038/scibx.2011.952

Published online Sept. 1, 2011

\section{REFERENCES}

1. Hsu, Y.-H. et al. J. Exp. Med.; published online Aug. 15, 2011; doi:10.1084/jem.20102234

Contact: Ming-Shi Chang, National Cheng Kung University, Tainan City, Taiwan e-mail: mschang@mail.ncku.edu.tw

2. Chen, W.-Y. et al. Arterioscler. Thromb. Vasc. Biol. 26, 2090-2095 (2006)

3. Chen, W.-Y. \& Chang, M.-S. J. Immunol. 182, 5003-5012 (2009)

4. Hsu, Y.-H. et al. Arthritis Rheum. 54, 2722-2733 (2006)

5. Simonet, W.S. et al. Cell 89, 309-319 (1997)

\section{COMPANIES AND INSTITUTIONS MENTIONED}

Ablynx N.V. (Euronext:ABLX), Ghent, Belgium

Amgen Inc. (NASDAQ:AMGN), Thousand Oaks, Calif. Daiichi Sankyo Co. Ltd. (Tokyo:4568; Osaka:4568), Tokyo, Japan GlaxoSmithKline plc (LSE:GSK; NYSE:GSK), London, U.K. National Cheng Kung University, Tainan City, Taiwan Novo Nordisk A/S (CSE:NVO; NYSE:NVO), Bagsvaerd, Denmark Tokyo Medical University, Tokyo, Japan 